\title{
Understanding how distance to facility and quality of care affect maternal health service utilization in Kenya and Haiti: A comparative geographic information system study
}

\author{
Xing Gao, ${ }^{1}$ David Wayne Kelley ${ }^{2}$ \\ ${ }^{1}$ School of Public Health, University of California-Berkeley, Berkeley, CA; ${ }^{2}$ Department of Geography \\ and Environmental Studies, University of St. Thomas, St. Paul, MN, USA
}

\begin{abstract}
In 2000, the United Nations established eight Millennium Development Goals (MDG) to combat worldwide poverty, disease, and lack of primary education. Goal number five aimed to reduce the maternal mortality ratio by three quarters and provide universal access to reproductive healthcare services by 2015 . While there has been some progress, MDG 5 fell far short of target goals, highlighting the necessity of further improvement in global maternal health. Using Geographic Information Systems (GIS), this study aims to understand how distance to facility and quality of care, which are components of access, affect maternal service utilization in two of the world's poorest countries, Haiti and Kenya. Furthermore, this study examines how this relationship may change or hold between urban and rural regions. Data from the United States Agency for International Development Demographic and Health Survey and Service Provision Assessment were linked spatially in a GIS model, drawing comparisons among distance to facility, quality of care, and maternal
\end{abstract}

Correspondence: David Wayne Kelley, Department of Geography and Environmental Studies, University of St. Thomas, 2115 Summit Ave., JRC432, St. Paul, Minnesota 55105, USA.

Tel.: +1.651.962.5569 - Fax: +1.651.962.5741.

E-mail: dwkelley@stthomas.edu

Key words: Geographic Information Systems (GIS); Antenatal care; Maternity care; Health service utilization; Maternal mortality ratio.

Contributions: the authors contributed equally.

Conflict of interest: the authors declare no potential conflict of interest.

Funding: none.

Received for publication: 14 March 2018.

Revision received: 4 January 2019.

Accepted for publication: 12 January 2019.

CC Copyright X. Gao and D.W. Kelley, 2019

Licensee PAGEPress, Italy

Geospatial Health 2019; 14:690

doi:10.4081/gh.2019.690

This article is distributed under the terms of the Creative Commons Attribution Noncommercial License (CC BY-NC 4.0) which permits any noncommercial use, distribution, and reproduction in any medium, provided the original author(s) and source are credited. health service utilization. Results show that in both rural and urban regions, access to maternal health service and maternal health service utilization share a similar spatial pattern. In urban regions, pockets of maternal health disparities exist despite close distance to facility and standard quality of care. In rural regions, there are areas with long distances to facilities and low quality of care, resulting in poor maternal service usage. This study highlights the usefulness of GIS as a tool to evaluate disparities in maternal healthcare provision and usage.

\section{Introduction}

\section{Maternal health}

The effort to address maternal health outcomes solidified women's wellbeing as a vital part of development. Maternal deaths are preventable; there are cost-effective, simple interventions available to ensure safe pregnancies. The World Health Organization (WHO) recommends universal access to skilled care during pregnancy, timely interventions for complications, access to family planning, and services for reproductive health, especially in vulnerable populations (United Nations, 2015b). Furthermore, strengthening the monitoring and evaluation of evidence-based interventions, as well as improving data collection on health outcomes, are both vital to ensuring accurate measurement of progress (United Nations, 2015b).

Maternal mortality, defined as the death of women while pregnant or within 42 days of pregnancy termination from any cause related to or aggravated by pregnancy or its management, continues to be a challenge in public health (WHO, 2014). At the beginning of the 21 st century, 189 countries endorsed the United Nation's Millennium Declaration, which outlined eight goals deemed critical to future global development. Millennium Development Goal (MDG) 5: Improve Maternal Health sought to: i) reduce the maternal mortality ratio (MMR) by three quarters between 1990 and 2015, and ii) achieve universal access to reproductive health services by 2015. MMR is one of the most important and globally recognized indicators for measuring the state of a country's health system.

Globally, through the efforts of MDG5, the maternal mortality ratio dropped by $45 \%$ between 1990 and 2013, from 380 to 210 deaths per 100,000 live births (United Nations, 2015b). However, this still fell short of the three-quarters goal outlined in MDG Target 5.A. To achieve a 75\% reduction in MMR between 1990 and 2015, a 5.5\% annual decline in MMR would have been required from 1990 onward. However, the annual percentage decline in MMR globally between 1990 and 2008 was only $2.3 \%$ 
(WHO, 2014). Furthermore, such progress is unevenly distributed on both global and national scales. Less-developed regions face MMR fourteen times higher than that of developed regions. Additionally, maternal deaths are concentrated in Sub-Saharan Africa (62\%) and Southern Asia (24\%), followed by Oceania and the Caribbean (United Nations, 2015b). Thirty countries with an MMR $\geq 100$ in 1990 made insufficient or no progress, including 23 from sub-Saharan Africa (WHO, 2014). Understanding the complex factors that contribute to maternal mortality continues to be a prominent challenge. Within countries, the urban and rural divisions also see unequal distribution of maternal health improvement. Traditionally, rural areas have had poorer health access for women. Progress in this inequity has been slow; in less-developed regions, the urban-rural gap in the proportion of births attended by a skilled health professional barely narrowed from $33 \%$ to $31 \%$ between 2000 and 2012 (United Nations, 2015b). As urbanization occurs at an unprecedented rate, the comparative advantages cities enjoy over their rural counterparts are also diminishing (United Nations, 2014). Within urban areas, huge disparities in access to prenatal care and skilled birth attendance exist between rich neighborhoods and impoverished slums (Fotso et al., 2009; Mills et al., 2009; Save the Children, 2015). Therefore, further research is needed to understand the factors that contribute to maternal mortality, especially with a focus on ameliorating the unequal distribution of improvement. To improve maternal health, the WHO recommends that all women should obtain four antenatal visits during the course of their pregnancies (United Nations, 2015b). Furthermore, skilled birth attendance plays a critical role in reducing maternal and newborn mortality and morbidity (WHO, 2004). A skilled birth attendant with proficient training and a functional health system at their disposal can offer interventions to prevent or treat life-threatening complications such as heavy bleeding, or refer a patient to a higher level of care when necessary. Increased access to antenatal visits and skilled attendance at delivery are key interventions that have proven to be effective to improve maternal health outcomes (WHO, 2014; Salehi and Ahmadian, 2017).

This study focuses on rural and urban areas in Kenya and Haiti, two countries that struggle with combating maternal mortality in urban regions and a lack of access to services in rural areas. Among the 23 sub-Saharan African countries with $M M R \geq 100$ in 1990, Kenya still struggles with maternal mortalities of 488 per 100,000 live births, with some regions reporting MMRs of over 1,000 per 100,000 live births (KNBS, 2010). In the western hemisphere, Haiti is included in this list of countries that have made insufficient progress (300 maternal deaths per 100,000 live births). Both Kenya and Haiti fell short of their goals to decrease MMR by $75 \%$ as outlined in MDG Target 5.A. Kenya's decline from 1990 to 2015 averaged just 26\% while Haiti averaged 43\% (United Nations, 2015a). Annual decreases for Kenya during this time period ranged from a low of $-2.72 \%$ (an increase) in 1996 to $4.42 \%$ in 2010 , with a 15 -year average of $1.16 \%$. For Haiti, annual decreases ranged from a low of $-0.81 \%$ (an increase) in 2012 to a high of $5.14 \%$ in 2011 , with a 15 -year average of $2.18 \%$.

Additionally, with an annual rate of change in urban populations of $1.7 \%$ in Kenya and $2.4 \%$ in Haiti, these countries serve as case studies for countries combating maternal mortality coupled with rapid growth of urban slums (United Nations, 2014). Due to the differences in their history of healthcare, socio-economic context, and geographic landscape, these countries also face unique obstacles. For example, Haiti exemplifies the relationship between distance, quality of care, and maternal health in a post-disaster set- ting (Amibor, 2013). Kenya, on the other hand, struggles with maternal health disparities within urban areas. Both accessibility and availability influence access. Often, people consider geographical and non-geographical factors when they make decisions about health service use (Cromley and McLafferty, 2011). This study aims to offer a comparative exploration of how the effects of distance and quality of care influence maternal health outcomes in both Kenya and Haiti in the hopes that this exploration can offer insights into this relationship in other similar settings. Recent, comprehensive datasets of demographic and health surveys and facility provision assessments for these countries are available for free download from the United States Agency for International Development (USAID) Demographic and Health Survey (DHS) website (USAID, 2015). The original contribution of this paper is that it links country-specific USAID DHS and Service Provision Assessment (SPA) files to locational data to identify spatial deficiencies in maternal health care delivery. Two least developed countries (LDCs), one in sub-saharan Africa (Kenya) and the only one in the Americas (Haiti), are used to demonstrate the practicality and efficacy of linking health care metrics to spatial data for the purpose of identifying underserved populations and service areas.

\section{Study areas: Kenya and Haiti}

In 2013, Kenya experienced an estimated 400 maternal deaths per 100,000 live births (WHO, 2014). In an effort to address this concern, the government has abolished user fees in all public maternity hospitals and clinics. Although in practice, most women pay a small fee (Mason et al., 2015). Furthermore, mothers are being encouraged to deliver in the nearest maternity facility with a skilled health worker (UNDP, 2015). Kenya utilizes a 6-tier health system, with the basic level staffed by community health workers, followed by tiers 2 and 3, which are dispensaries and health centers that provide the majority of health services for pregnant women (Mason et al., 2015). According to the 2014 Kenyan demographic and health survey, there has been a positive trend in antenatal care with a percentage increase of mothers reporting antenatal visits to a health professional from $88 \%$ to $96 \%$ between 2003 and 2014 . Furthermore, the percentage of births attended by a skilled provider (62\%) and percentage occurring in health facilities $(61 \%)$ each increased by a similar magnitude (20\%) from 2003 to 2014. Despite this progress, there is still room for improvement, especially in addressing the maternal health of marginalized populations (United Nations, 2015b).

In Kenya, compared to rural residents, urban residents were more likely to deliver in a facility, highlighting the challenges in rural area access (Kitui et al., 2013). Half of rural Kenyan mothers received skilled care or delivered in a health facility, compared to $82 \%$ of urban mothers (KNBS, 2015). However, the urban slums in major cities such as Nairobi also harbor challenging conditions for mothers. In a study conducted from 2003-2005, the maternal mortality ratio for the two Nairobi slums was nearly double the national average (Mills et al., 2009). Another study found that although $70 \%$ of slum residents delivered at health facilities, only about $48 \%$ delivered in facilities with at least a minimum standard of care as measured by the availability of six key emergency obstetric procedures (Fotso et al., 2009). Certain socioeconomic groups bear a disproportionate burden of poor maternal health outcomes. Studies also found that individual-level factors correlated with receiving antenatal care and delivering with attendance. Wealth, education level, employment, and pregnancy parities usually correlated positively, while number of past births and limited 
disposable time (time left to the individual after family responsibilities have been met) correlated negatively with maternal health outcomes (McCray, 2004; Kitui et al., 2013; Rossier et al., 2014; KNBS, 2015).

Haiti also faces significant challenges in terms of maternal health. Although facility delivery increased from $25 \%$ in 2005 2006 to $36 \%$ in 2012, with the portion of births attended by a skilled attendant increasing to $37 \%$ between $1990-2010$, the maternal mortality rate was 380 per 100,000 in 2013 (Babalola, 2014). Haitian women in both rural and urban settings have unsafe pregnancies. In rural Haiti, women cited the cost of care, heavy dependence on their husbands, and dissatisfaction with the services as reasons for their delay in seeking care during pregnancy (White et al., 2006). Moving from individual to contextual factors, one study found that road conditions and access to a nearby facility govern rural care seeking during pregnancy, while delivery services are influenced by geography and distance from the nearest hospital (Gage and Guirlene Calixte, 2006).

Urban Haiti witnesses poor maternal health as well. Port-auPrince, Haiti, has one of the largest gaps globally in healthcare coverage between the richest and poorest $20 \%$ of mothers and children. In Port-au-Prince, the poorest $20 \%$ have a prenatal care rate of $36 \%$, compared to $87 \%$ in the richest $20 \%$ (Save the Children, 2015 ). Furthermore, only $18 \%$ of the poorest urban residents had a skilled birth attendant, compared to $72 \%$ of the richest population (Save the Children, 2015). Since 70\% of Haiti's urban population resides in slums, these statistics reveal a staggering struggle to achieve equal progress in maternal health outcomes. In 2008, the Haitian government, recognizing the pressing issue of maternal deaths, implemented the Soins obstétricaux gratuits scheme, which provides free access to obstetric health care (Amibor, 2013).

\section{Utilization of geographic information systems}

The use of quality maternal health services is central to further progress in reducing maternal deaths. Access has long been recognized as a significant factor in people's utilization of health services (Higgs, 2009). Past researchers have explained access in terms of entry into the health system (Donabedian, 1973; Aday and Anderson, 1975). Furthermore, Penchansky and Thomas (1981) identified five key components of access: availability, accessibility, accommodation, affordability, and acceptability. The first two components can be defined in spatial terms, with availability denoting adequacy of healthcare provision and accessibility referring to geographic impedance between healthcare supply and demand. Distance-based measures are increasingly utilized in geographic access studies because it considers the variation in spatial access within administrative boundaries (Cromley and McLafferty, 2011). Distance from household to facility has been known to affect the uptake of maternal healthcare services (Tanser et al., 2006; Hounton et al., 2008; Gabrysch and Campbell, 2009). This distance-decay effect affects not just utilization of health care services but health outcomes (Feikin et al., 2009; Gething et al., 2012). Recent studies in the Sub-Saharan African countries of Burkina Faso, Uganda, and the Democratic Republic of the Congo (formerly Zaire) found that increased perinatal and infant mortality rates were associated with distance to facility greater than 5 kilometers (Aoun et al., 2015).

There are several methods used to create distance-based measurements. For example, measures based on Euclidean distance, service area analysis, and origin-destination surveys can all be used as surrogate indicators for access (WHO, 2001; Masters et al.,
2013). However, physical distance may not be the most significant measurement of access since it does not account for travel time or cost, difficult measures to assess for any country given the varied road conditions, season, natural obstacles, and available transportation (Rutherford et al., 2010; Kitui et al., 2013). More sophisticated models of spatial interaction, such as the gravity model and its related two-step floating catchment area (2SFCA) method, take into account how people may choose a facility based on service availability or the quality of care provided (Luo and Wang, 2003; Yao et al., 2013)

Geographic Information Systems (GIS) can play an important function in linking individual data with spatial and contextual data. One shortcoming of previous spatial studies were surveys that collected data on individual service use rarely also collected data on the health services available to these individuals (Gage and Guirlene Calixte, 2006). In some cases, studies may work around this by collecting both sets of data on a small scale, or by conducting large-scale household surveys with limited, additional facility data. Contrarily, geographic studies usually evaluate access without controlling for level of quality within the facilities (Gabrysch et al., 2011). This explains why there have been limited studies concerning how the level of service quality at facilities affects utilization of maternal care (Hounton et al., 2008). A similar study has used GIS to link demographic and health data with service provision assessment data in rural Zambia through geo-coordinates (Gabrysch et al., 2011).

This study utilizes GIS and a 2SFCA model to determine access scores for maternal health service utilization based upon the distance to a facility and its quality of care. This examination draws a comparison between urban and rural regions of Kenya and Haiti.

\section{Materials and Methods}

\section{Data}

The data used in this study were collected from the USAID Demographic and Health Survey program (USAID, 2015). The first type of survey accessed was the DHS, which has three sections consisting of a household questionnaire, an individual questionnaire, and biomarker data. The DHS utilizes an individual questionnaire for women with a two-stage sampling design; the chosen clusters are representative of the national populations. The Haiti DHS survey was conducted in 2012, the Kenya survey in 2014.

The second type of survey accessed from the DHS site was the SPA, which examines the availability of health services at different types of facilities including human resources, infrastructure, and supplies; the types of support services and processes available; quality of services as compared to accepted standards; and client understanding, satisfaction, and perceptions of care. Haiti SPA surveys were compiled in 2010, Kenya surveys in 2013. The standard SPA questionnaire includes ten key service areas, ranging from facility infrastructure to malaria diagnostic and treatment services. The data of interest in this study were taken from the Facility Data file, which consolidated results from the inventory questionnaire.

Data were converted from the native SAS format to a commadelimited text file for use in a GIS. 


\section{Country specific study areas}

For the purpose of this research, the urban study areas were determined by the DHS and SPA surveys. In Haiti, the DHS survey region denoted Aire Métropolitaine, which includes Port-au-Prince and its surrounding urban communes, was used as the urban study area (Figure 1A and B). The region (department) denoted GrandAnse was used as the rural study area because of its low population density vis-à-vis its size. In Kenya, the DHS survey region denoted Nairobi refers to the urban study area and the Western province defines the rural study area, selected based on its distance from the capitol and generally low living standards such as the lack of running water and electricity).

\section{Two-step floating catchment area model}

In this study, quality of facilities serves as a measurement of availability and distance serves as a measurement of accessibility. People are likely to travel farther for more specialized services, high-quality services, or larger facilities. When it comes to choosing a maternal care provider during delivery, features such as emergency obstetric care or range of services provided may contribute to the perceived quality of a facility. Based on the model, origins with low accessibility may be caused by a combination of being far from facilities or being near less attractive services. The model calculates the access score of each DHS survey cluster, which consists of groupings of households that participated in the survey. A resident's access score is calculated as the sum of connected facilities' quality values divided by the average distance to connected facilities. Connected facilities from a residence include all health care facilities within $10 \mathrm{Km}$ in rural areas (Yao et al., 2013) and $5 \mathrm{Km}$ in urban areas (Scott et al., 2013). Access scores are expressed as traditional service capacity ratios with a lower limit of zero (no access) to an unspecified upper limit (summary score based on a variable number of facilities and distances in each DHS cluster).

The model (Cromley and McLafferty, 2011) is defined as:

Access score $=\sum_{j} A_{j} / d_{i j}{ }^{\beta}$

where: $A_{j}=$ quality of the facility; $d_{i j}=$ the distance from residence $i$ (origin) to facility $j$ (destination); $\beta=$ exponent serving to model distance decay. Assigned value $=1$.

\section{Quality of the facility}

In this analysis, the quality of each facility $\left(A_{j}\right)$ was calculated based on a value generated through an index, with the indicators
A
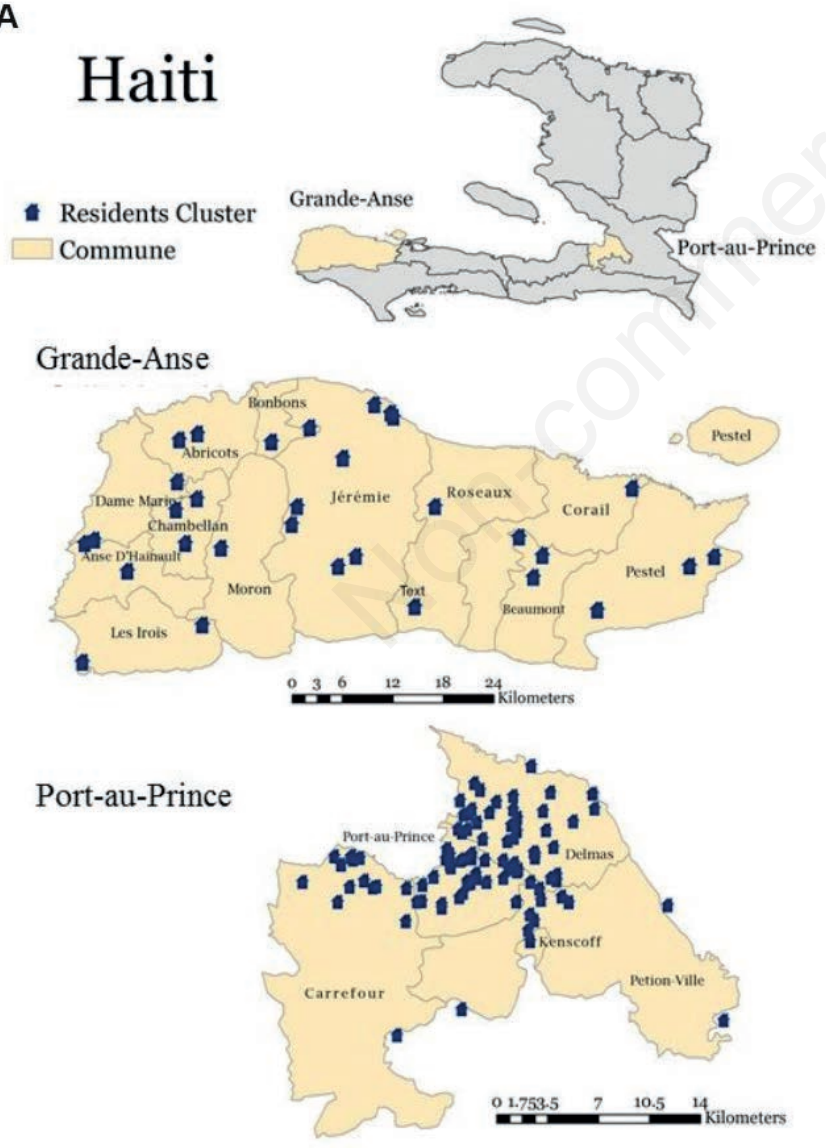

B

\section{Kenya}

- Residents Cluster Division
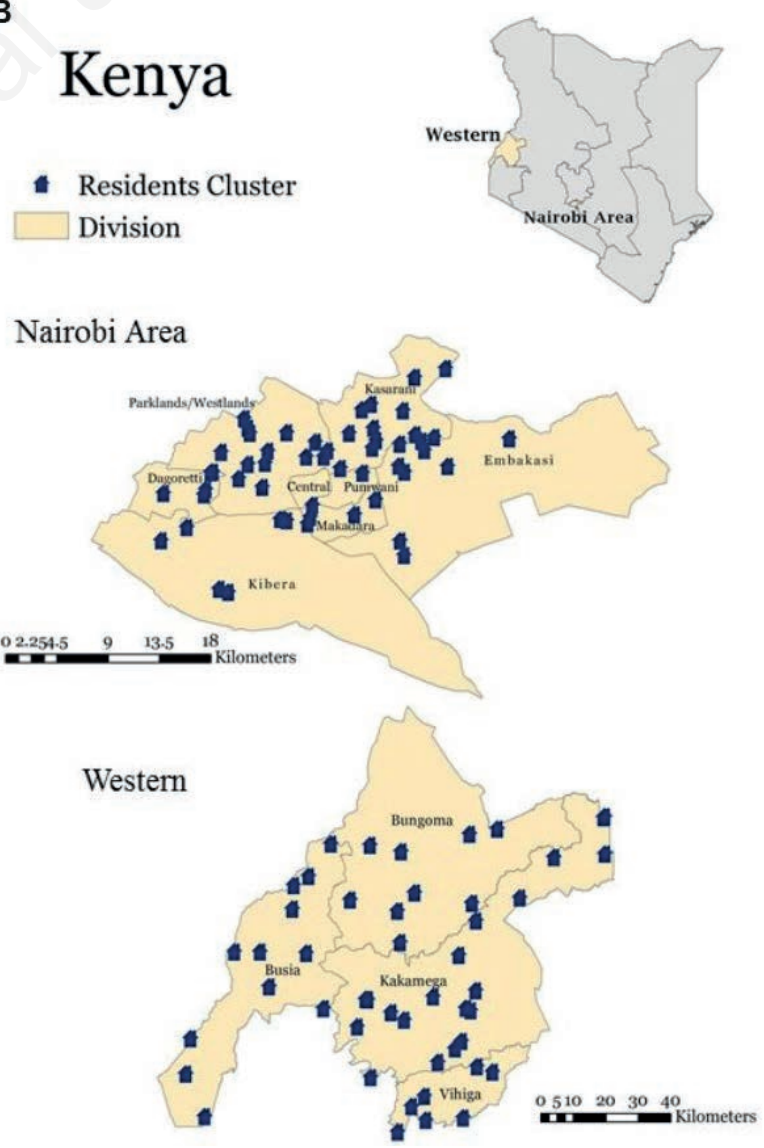

Figure 1. (A) Reference map: Haiti. Blue points indicate Demographic and Health Survey (DHS) clusters (origins i within the model). (B) Reference map: Kenya. Blue points indicate DHS clusters (origins i within the model); Nairobi slums are located primarily in the Kibera Division. 
shown in Table 1. The first six indicators describe basic emergency obstetric care. Two more indicators ( 7 and 8 ), in addition to the first six, together describe comprehensive emergency obstetric care (AMDD, 2003). Other indicators, such as the presence of a skilled birth attendant and the availability of ambulance services, were also taken into consideration. For each indicator, the facility was assigned a value of 1 if it satisfied the indicator and 0 if it did not. Zero was also assigned if the facility did not respond to the question or if the response was unknown or not sure. The only two non-binary indicators are the number of maternal beds and the number of delivery beds. The numeric values of those indicators were standardized into a value between 0 and 1 . Outlier values above 1 were rounded to 1 .

\section{Distance to facility}

Within the model, origins $i$ were defined as each DHS cluster (shown in blue in Figure 1A and B) and destinations $j$ were defined as the facilities. Each origin is connected to all facilities within a set range: 10 kilometers in rural areas and 5 kilometers in urban areas. Each origin's quality score was calculated based on the total quality scores of all connected facilities and the origin's average distance $(\mathrm{km})$ from all connected facilities. An interpolation method (inverse distance weighting, IDW) was used to model continuous geographic variation across the service area based on the calculated quality value. $\beta$ value was 1 .

\section{Maternal health service utilization}

Maternal health service utilization was calculated for each DHS cluster and then interpolated using IDW to create a continu- ous geographic model that showed variation across the study areas. The measurements used to map maternal health service utilization were the number of antenatal visits during the most recent pregnancy and the percentage of the cluster that delivered in a facility.

\section{Statistical analysis}

Using the results from the 2SFCA model analysis and maternal health service utilization mapping, the relative impact of geographic access compared to other individual level factors on maternal health service utilization was evaluated in a regression analysis applied to each DHS cluster. The individual factors are listed in Table 2.

\section{Results}

In rural Haiti (Figure 2 and Table 3), the access scores produced similar patterns of maternal health services for antenatal visits and facility delivery. The southwestern communes (Moron, Les Trois, Jérémie) have low access scores, which also correlate with fewer antenatal visits and a lower rate of facility delivery. The facilities are sparsely located in these areas. On the other hand, in the eastern region, although the facilities are present, access and service utilization remained low. The average access score is 2.49 and the average antenatal visit number is 4.15 . In the Grande-Anse department, the level of education positively influences the number of antenatal visits. The level of dependency on other household members in healthcare decisions negatively relates to the chance of

Table 1. Facility quality indicators.

Basic Emergency Obstetric Care (provided within the last three months)

1. Administer parenteral antibiotics (intravenous or by injection)

2. Administer parenteral oxytocic drugs

3. Administer parenteral anticonvulsants

4. Perform manual removal of placenta

5. Perform removal of retained products

6. Perform assisted vaginal delivery (vacuum extraction, forceps delivery)

Comprehensive Emergency Obstetric Care (provided within the last 3 months) 7. Perform surgery (Cesarean section)

8. Perform blood transfusion

Other indicators

9. National guidelines for IMPAC

10. National guidelines for CEmOC

11. Skilled birth attendant on call $24 / 7$

12. Fee for normal delivery

13. Fee for C-section

14. Ambulance availability

15. Number of Maternal beds

16. Number of Delivery beds

IMPAC, Integrated Management of Pregnancy and Childbirth; CEmOC, Comprehensive Emergency Obstetric Care.

Table 2. Individual level factors.

\begin{tabular}{|c|c|}
\hline Factor & Explanation \\
\hline Age Group & Respondent's age classified into groups \\
\hline Education Level & No education, primary education, secondary education, or higher education \\
\hline Employment & Respondent's current employment status \\
\hline Dependency & $\begin{array}{l}\text { If respondent, respondent and other household members, or other household } \\
\text { members have the final say in the respondent's healthcare decision }\end{array}$ \\
\hline Pregnancy undesirability & If the pregnancy was wanted at the time, wanted in the future, or not at all \\
\hline Number of births & How many births the respondent has experienced \\
\hline
\end{tabular}




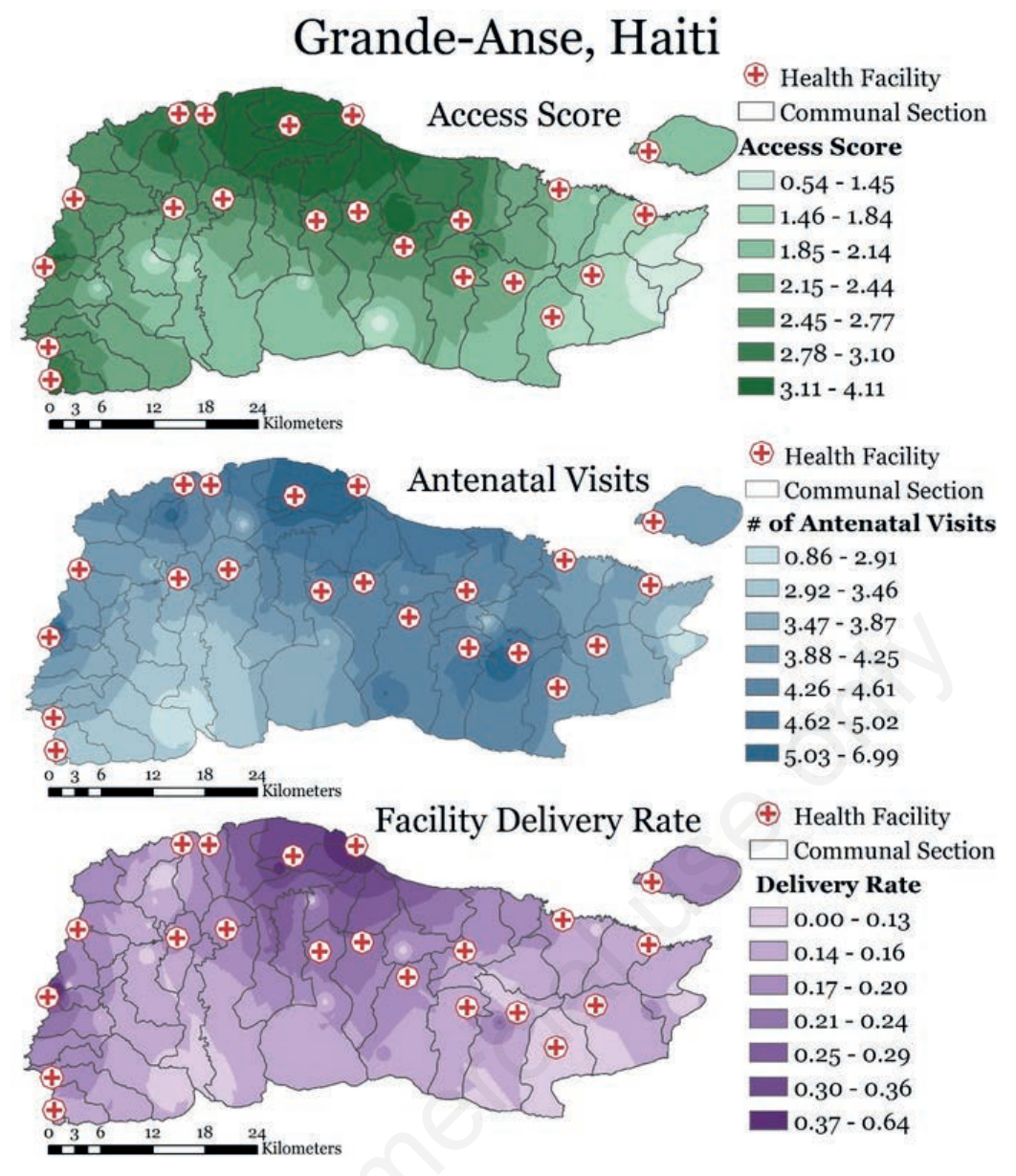

Figure 2. Results for Rural Haiti, Grande-Anse department.

Table 3. Regression analysis for rural and urban Haiti.

\begin{tabular}{|c|c|c|c|c|}
\hline \multicolumn{3}{|c|}{ Antenatal Visit } & \multicolumn{2}{|c|}{ Facility Delivery } \\
\hline \multicolumn{5}{|c|}{ Rural Haiti } \\
\hline Factor & Coefficient & P-Value & Coefficient & P-Value \\
\hline Access Score & 0.34 & 0.13 & 0.03 & 0.33 \\
\hline Age Group & 0.37 & 0.90 & 0.33 & 0.37 \\
\hline Education & 1.07 & $0.09 *$ & 0.10 & 0.19 \\
\hline Employment & 0.08 & 0.96 & 0.04 & 0.82 \\
\hline Dependency & -0.20 & 0.79 & -0.18 & $0.07^{*}$ \\
\hline Pregnancy undesirability & -1.62 & 0.10 & -0.12 & 0.32 \\
\hline Number of births & 0.04 & 0.93 & 0.04 & 0.57 \\
\hline \multicolumn{5}{|c|}{ Urban Haiti } \\
\hline Factor & Coefficient & P-Value & Coefficient & P-Value \\
\hline Access Score & 0.01 & 0.20 & 0.00 & 0.11 \\
\hline Age Group & -0.37 & 0.18 & 0.13 & 0.54 \\
\hline Education & 0.65 & $0.01^{* *}$ & 0.19 & $0.00 * * *$ \\
\hline Employment & 0.37 & 0.50 & -0.05 & 0.57 \\
\hline Dependency & 0.30 & 0.37 & 0.02 & 0.66 \\
\hline Pregnancy undesirability & -1.70 & $0.01^{* *}$ & 0.00 & 0.11 \\
\hline Number of births & 0.03 & 0.90 & -0.01 & 0.80 \\
\hline
\end{tabular}

${ }^{*} \mathrm{P}<0.1,{ }^{* *} \mathrm{P}<0.05,{ }^{* * * \mathrm{P}<0.01}$. 
delivery in a facility. Approximately $18.8 \%$ of deliveries took place in a facility in Grande-Anse. While access score positively correlates with the use of either maternal health service, the relationships are not significant.

In urban Haiti (Figure 3 and Table 3), the access scores are the highest of all study areas. The highest access scores are concentrated in the central part of Port-au-Prince and decrease toward the outer areas. The southeastern communes (Petion-Ville) have fewer antenatal visits and lower facility delivery rates. Although access scores are relatively high in the center area, there are small pockets of low service utilization rates within Port-au-Prince. The average access score is 43.1 and the average antenatal visit number is 5.8 . Approximately $60 \%$ of deliveries took place in a facility. The level of education has a statistically significant, positive relationship with both the number of antenatal visits and the rate of facility delivery. Additionally, pregnancy undesirability correlates negatively with the number of antenatal visits.

In rural Kenya, access is the lowest out of all study areas
(Figure 4 and Table 4). The access score map shows that access is low in the outer southwestern and upper eastern areas. Whereas the access map shows high access in the center of the province, contrarily, the maternal health service utilization map shows poorer service usage rate in the center. The average access score is 0.68 and the average antenatal visit number is 3.6. Approximately $36 \%$ of deliveries took place in a facility. Rural Kenya is the only study area where the access score significantly correlates to maternal health outcome of a facility delivery. Additionally, age group has a negative correlation with health service usage; as women age, they are less likely to receive antenatal care and deliver in a facility. Level of education has a statistically significant, positive relationship with the rate of facility delivery. Additionally, pregnancy undesirability correlates negatively with facility delivery rate.

In urban Kenya (Figure 5 and Table 4), access scores are high in the center and decrease toward the outer areas. In particular, Kibera, an urban slum area located in eastern Nairobi, has a low access score and poor maternal health service utilization.

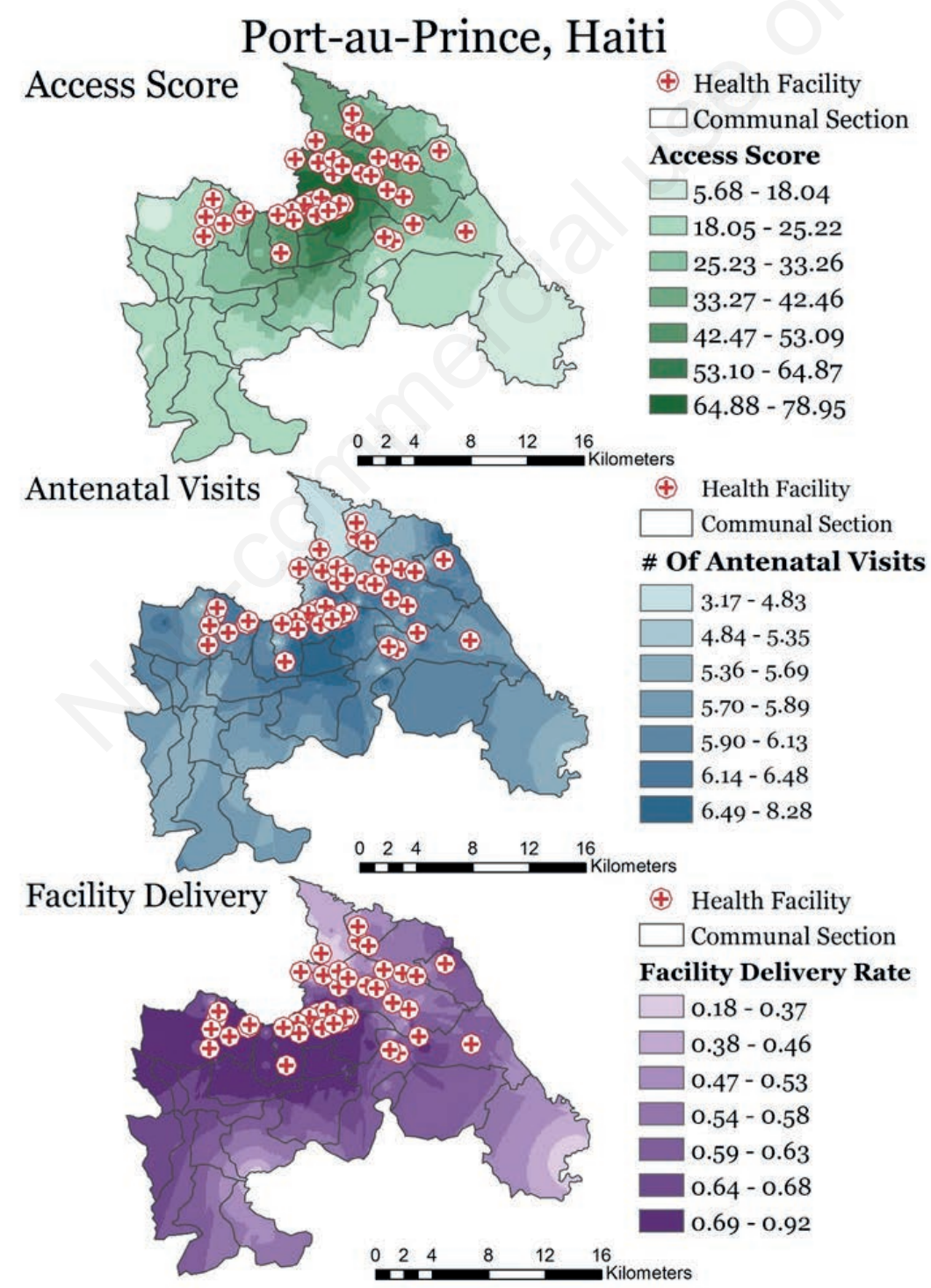

Figure 3. Results for Urban Haiti, Port-au-Prince commune. 
Additionally, there are pockets of low service utilization in central Nairobi where facilities are concentrated. The western area of Kibera has a low access score, correlated with low rates of maternal health service utilization. The average access score is 4.7 and the average number of antenatal visits is 5.3. Approximately $90 \%$ of deliveries took place in a facility. In urban Kenya, the level of education has a statistically significant relationship with both the number of antenatal visits and facility delivery rate. Employment status also relates positively with the number of antenatal visits.

Table 4. Regression analysis for rural and urban Kenya.

\begin{tabular}{|c|c|c|c|c|}
\hline \multicolumn{3}{|c|}{ Antenatal Visit } & \multicolumn{2}{|c|}{ Facility Delivery } \\
\hline \multicolumn{5}{|c|}{ Rural Kenya } \\
\hline Factor & Coefficient & P-Value & Coefficient & P-Value \\
\hline Access Score & -0.09 & 0.36 & 0.06 & $0.02 * *$ \\
\hline Age Group & -2.40 & $0.07 *$ & -1.00 & $0.00^{* * *}$ \\
\hline Education & 0.23 & 0.37 & 0.22 & $0.00^{* * *}$ \\
\hline Employment & -0.10 & 0.83 & -0.07 & 0.58 \\
\hline Dependency & 0.34 & 0.29 & -0.13 & 0.13 \\
\hline Pregnancy undesirability & -0.31 & 0.51 & -0.25 & $0.06^{*}$ \\
\hline Number of births & -0.26 & 0.31 & -0.03 & 0.70 \\
\hline \multicolumn{5}{|c|}{ Urban Kenya } \\
\hline Factor & Coefficient & P-Value & Coefficient & P-Value \\
\hline Access Score & -0.06 & 0.32 & 0.01 & 0.18 \\
\hline Age Group & 0.74 & 0.58 & -0.03 & 0.79 \\
\hline Education & 0.08 & $0.00 * * *$ & 0.08 & $0.00 * * *$ \\
\hline Employment & 1.65 & $0.02^{* *}$ & -0.11 & 0.13 \\
\hline Dependency & 0.25 & 0.68 & -0.01 & 0.81 \\
\hline Pregnancy undesirability & -0.25 & 0.67 & -0.07 & 0.22 \\
\hline Number of births & -0.49 & 0.22 & -0.04 & 0.28 \\
\hline
\end{tabular}

${ }^{*} \mathrm{P}<0.1,{ }^{* * \mathrm{P}}<0.05,{ }^{* * * \mathrm{P}<0.01}$.

\section{Western Province, Kenya}

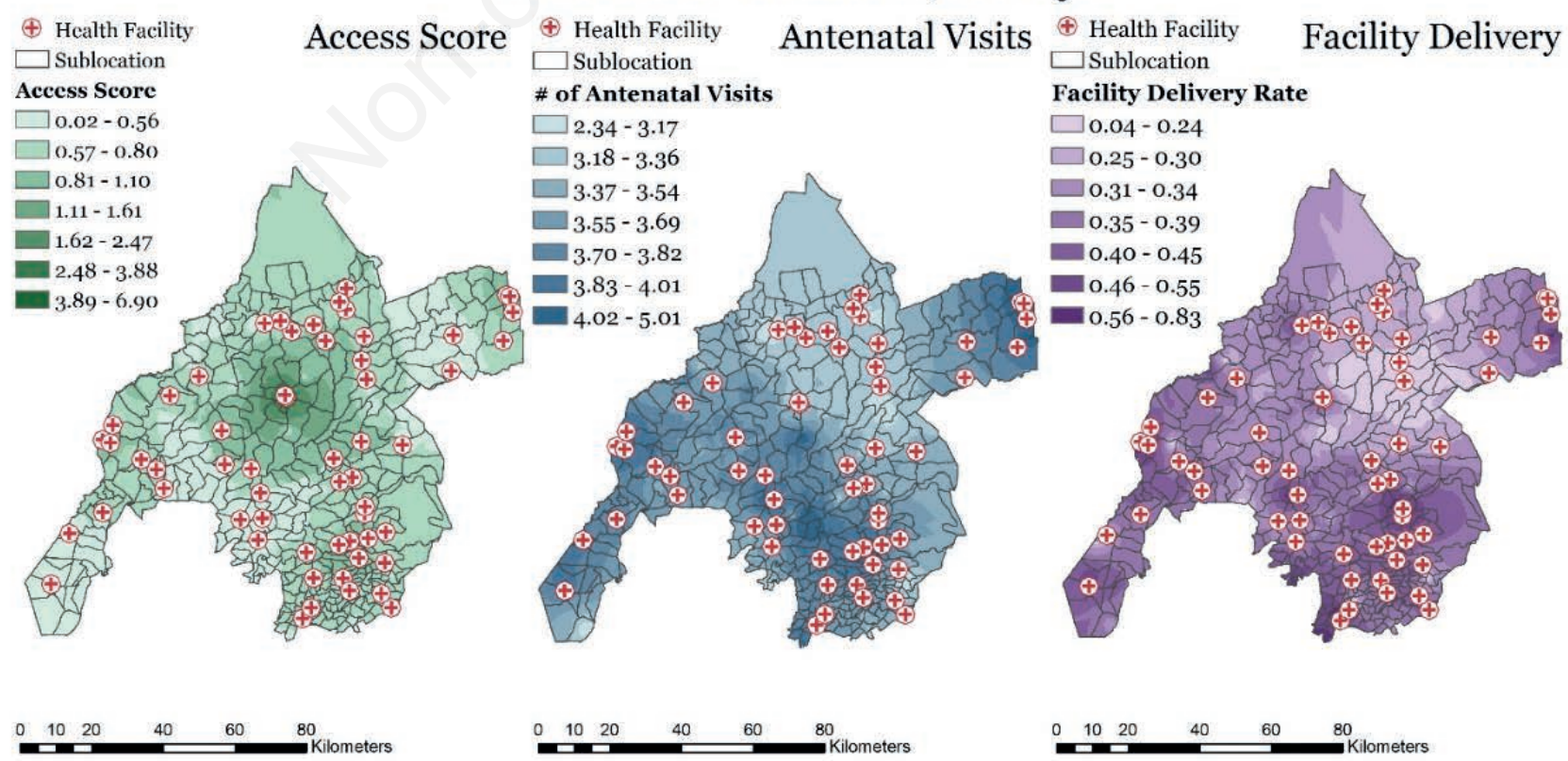

Figure 4. Results for Rural Kenya, Western province. 


\section{Discussion}

Using Haiti and Kenya as case studies, this research examined the effects of distance to facility and quality of care on maternal health services utilization in urban and rural regions. Overall, access score, which considers both distance and quality of the facilities, has a significant relationship with both maternal services utilization. This is shown by the similarities between the spatial patterns of access scores and maternal services utilization. Several themes emerged from the comparison between access and maternal health outcome maps. First, in urban regions, although access is high in the central area, several pockets of poor health outcomes are also located in the same area, confirming the need to address disparities within cities (Save the Children, 2015). Second, in rural areas, facilities are sparsely located in some areas, leading to low access. However, low rates of health service usage can occur in areas with few or many facilities. Overall, access score maps for all study areas overlap with the health service utilizations maps, with stronger similarities in some areas than others.

The regression analysis revealed the importance of also considering socioeconomic factors. For example, the level of education consistently correlates positively with health service usage across study areas. Furthermore, pregnancy undesirability, dependency, and age group all negatively correlate with maternal health service utilization. These socioeconomic factors have been welldocumented to predict a mother's utilization of health services (Fotso et al., 2009; Babalola, 2014). For example, studies cited dependency on a husband or relative as one of the reasons why rural Haitian mothers did not deliver in a facility (White et al., 2006). Access score only holds a statistically significant relationship with health service in one study area (rural Kenya). Comparatively, socioeconomic factors seem to influence utilization of maternal health services strongly.
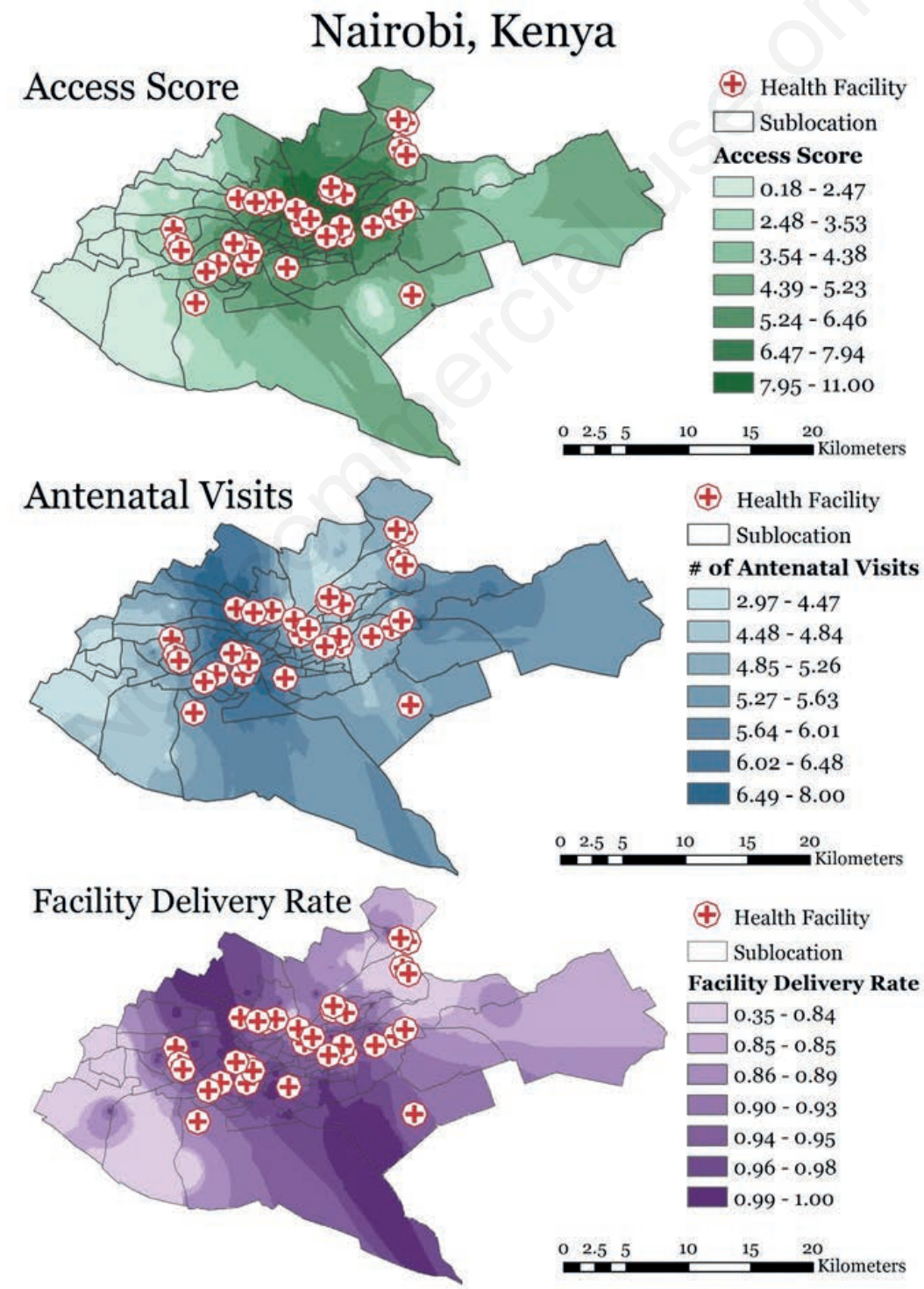

Figure 5. Results for Urban Kenya, Nairobi area. 


\section{Conclusions}

This study demonstrated the usefulness of GIS as a tool to promote health services utilization. Its ability to link the DHS and SPA datasets together can be translated to many other topics in public health involving the geographic aspect of access. Furthermore, mapping access and service utilization rates can help identify geographic areas that need more attention to promote maternal health equity among all populations. By identifying low access areas, the results of this study can help guide further, more comprehensive studies on the utilization of maternal health services in settings with limited resources and challenges in improving maternal health. The methods can also be applied to other LDCs or other health care delivery issues monitored in the DHS and SPA data in addition to maternal health. The benefit of this approach is that inefficiencies in health care delivery systems can be identified and resource allocation improved. The methods are applicable to both rural and urban populations and facilities.

Post-2015, Sustainable Development Goals build upon progress made with MDG5 and strive to reduce MMR to no more than 70 deaths per 100,000 live births by 2030 (UN Sustainable Development Summit, 2015). This continued focus on maternal health can benefit from the utilization of spatial interpolation to link comprehensive surveys. This linkage will aid the identification of areas with poor maternal health outcomes. For example, in areas where access is high but utilization rate is low, improvement of maternal health should focus on promoting utilization through education. Contrarily, areas with low access need more resources in terms of maternal service provision. By understanding the relationship between access and utilization, this study provides insights that can be used to improve maternal health in many settings.

\section{References}

Aday LA, Andersen R, 1975. Development of indices of access to medical care. Health Administration Press, University of Michigan, Ann Arbour, USA.

Amibor P, 2013. What will it take to maintain the maternal and child health gains made in Haiti prior to the 2010 earthquake?: An analysis of past progress, trends, and the prospects for the realization of the United Nations Millennium Development Goals 4 and 5. Matern Child Health J 17:1339-45.

Averting Maternal Death and Disability Program (AMDD), 2003. Using the UN process indicators of emergency obstetric services. Available from http://www.amddprogram.org/v1/resources/UsingUNIndicator sQA-EN.pdf.

Aoun N, Matsuda H, Sekiyama M, 2015. Geographical accessibility to healthcare and malnutrition in Rwanda. Social Sci Med 130:135-45.

Babalola SO, 2014. Factors associated with use of maternal health services in Haiti: a multilevel analysis. Rpsp Rev Panam Salud Pública 36:1-9.

Cromley E, McLafferty S, 2011. GIS and Public Health, 2nd ed. Guilford Press, New York, USA.

Donabedian A, 1973. Aspects of medical care administration: specifying requirements for health care. Harvard University Press, Cambridge, MA, USA.
Feikin DR, Nguyen LM, Adazu K, Ombok M, Audi A, Slutsker L, Lindblade KA, 2009. The impact of distance from a peripheral health facility on pediatric health utilization in rural Western Kenya. Trop Med Int Health 14:54-61.

Fotso J-C, Ezeh A, Madise N, Ziraba A, Ogollah R, 2009. What does access to maternal care mean among the urban poor? Factors associated with use of appropriate maternal health services in the slum settlements of Nairobi, Kenya. Matern Child Health J 13:130-7.

Gabrysch S, Cousens S, Cox J, Campbell OM, 2011. The Influence of distance and level of care on delivery place in rural Zambia: a study of linked national data in a geographic information system. PLoS Med 8:e1000394.

Gabrysch S, Campbell O, 2009. Still too far to walk: literature review of the determinants of delivery service use. BMC Pregnancy Childbirth 9:34.

Gage A, Guirlene Calixte M, 2006. Effects of the physical accessibility of maternal health services on their use in rural Haiti. Popul Stud 60:271-88.

Gething P, Johnson FA, Frempong-Ainguah F, Nyarko P, Baschieri A, Aboagye P, Falkingham J, Matthews Z, Atkinson P, 2012. Geographical access to care at birth in Ghana: a barrier to safe motherhood. BMC Public Health 12:991.

Higgs G, 2009. The role of GIS for health utilization studies: literature review. Health Serv Outcomes Res Methodol 9:84-99.

Hounton S, Chapman G, Menten J, DeBrouwere V, Ensor T, Sombie I, Meda N, Ronsmans C, 2008. Accessibility and utilization of delivery care within a skilled care initiative in rural Burkina Faso. Trop Med Int Health 13:44-52.

Kenya National Bureau of Statistics (KNBS) and ICF Macro, 2010. Kenya Demographic and Health Survey 2008-09. KNBS and ICF Macro, Calverton, Maryland, USA.

Kenya National Bureau of Statistics (KNBS), 2015. Kenya Demographic and Health Survey key indicators 2014. Available

from http://dhsprogram.com/pubs/pdf/PR55/PR55.pdf.

Kitui J, Lewis S, Davey G, 2013. Factors influencing place of delivery for women in Kenya: an analysis of the Kenya Demographic and Health Survey, 2008/2009. BMC Pregnancy Childbirth 13:1-10.

Luo W, Wang F, 2003. Measures of spatial accessibility to health care in a GIS environment: synthesis and a case study in the Chicago region. Environ Plan B: Plann Design 30:865-84.

Mason L, Dellicour S, Ter Kuile F, Ouma P, Phillips-Howard P, Were F, Laserson K, Desai M, 2015. Barriers and facilitators to antenatal and delivery care in western Kenya: a qualitative study. BMC Pregnancy Childbirth 15:26.

Masters SH, Burstein R, Amofah G, Abaogye P, Kumar S, Hanlon M, 2013. Travel time to maternity care and its effect on utilization in rural Ghana: A multilevel analysis. Social Sci Med 93:147-54

McCray T, 2004. An issue of culture: the effects of daily activities on prenatal care utilization patterns in rural South Africa. Social Sci Med 59:1843-55.

Mills S, Kyobutungi C, Ziraba A, Madise N, Ezeh A, 2009. Maternal mortality in the informal settlements of Nairobi city: what do we know? Reprod Health 6:1-8.

Penchansky R, Thomas JW, 1981. The concept of access: definition and relationship to consumer satisfaction. Med Care 19:127-40.

Rossier C, Muindi K, Soura A, Mberu B, Lankoande B, Kabiru C, 
Millogo R, 2014. Maternal health care utilization in Nairobi and Ouagadougou: evidence from HDSS. Glob Health Action 7:24351.

Rutherford M, Mulholland K, Hill P, 2010. How access to health care relates to under-five mortality in sub-Saharan Africa: systematic review. Trop Med Int Health 15:508-19.

Salehi F, Ahmadian L, 2017. The application of geographic information systems (GIS) in identifying the priority areas for maternal care and services. BMC Health Serv Res 17:482.

Save the Children, 2015. The urban disadvantage: state of the world's mothers 2015. Available from: http://www. savethechildren.org/atf/cf/\%7B9def2ebe-10ae-432c-9bd0df91d2eba74a\%7d/sowm 2015.pdf.

Scott S, Chowdhury ME, Pambudi ES, Qomariyah SN, Ronsmans C, 2013. Maternal mortality, birth with a health professional and distance to obstetric care in Indonesia and Bangladesh. Trop Med Int Health 18:1193-201.

Tanser F, Gijsbertsen B, Herbst K, 2006. Modelling and understanding primary health care accessibility and utilization in rural South Africa: An exploration using a geographical information system. Social Sci Med 63:691-705.

United Nations Development Programme (UNDP), 2015. Improve maternal health: Where we are? Maternal mortality in Kenya. Available from: http://www.ke.undp.org/content/kenya/en/ home/post-2015/mdgoverview/overview/mdg5.html

United Nations, 2014. World Urbanization Prospects: the 2014 revision highlights. Department of Economic and Social Affairs, Population Division, New York, USA.

United Nations, 2015a. Millennium Development Goals Indicators. Department of Economic and Social Affairs, United Nations Statistics Division. United Nations, New York, USA. Available from https://unstats.un.org/unsd/mdg/Data.aspx.
United Nations, 2015b. The Millennium Development Goals Report 2015. United Nations, New York, USA. Available from https://unstats.un.org/unsd/mdg/Resources/Static/Products/Pro gress2015/English2015.

United Nations Sustainable Development Summit, 2015. Goal 3: Ensure healthy lives and promote well-being for all at all ages. Available from http://www.un.org/sustainabledevelopment /health/

United States Agency for International Development (USAID), 2015. Demographic and Health Surveys; Haiti and Kenya. Available from http://dhsprogram.com/data/.

White K, Small M, Frederic R, Joseph G, Bateau R, Kershaw T, 2006. Health seeking behavior among pregnant women in rural Haiti. Health Care Women Int 27:822-38.

World Health Organization (WHO), 2001. Background paper for the technical consultation on effective coverage of health systems 27-29 August 2001, Rio de Janeiro, Brazil. WHO, Geneva, Switzerland.

World Health Organization (WHO), 2004. Making pregnancy safer: the critical role of the skilled attendant; a joint statement by WHO, ICM, and FIGO. WHO, Geneva, Switzerland. Available from http://whqlibdoc.who.int/publications/2004/ 9241591692.pdf.

World Health Organization (WHO), 2014. Trends in maternal mortality: 1990 to 2013, estimates by WHO, UNICEF, UNFPA, The World Bank and the United Nations Population Division. Department of Reproductive Health and Research, WHO, Geneva, Switzerland. Available from http://apps.who.int/iris/ bitstream/10665/112682/2/9789241507226 eng.pdf?ua=1.

Yao J, Murray AT, Agadjanian V, 2013. A geographical perspective on access to sexual and reproductive health care for women in rural Africa. Social Sci Med 96:60-8. 